\title{
Case Report: ALK Traslocated Pulmonary Sarcomatoid Carcinoma
} Alain Jonathan Gelibter*, MD, Marco Siringo, MD, Valerio Maria Napoli MD, Enrico Cortesi MD, Paolo Marchetti MD Division of medical Oncology “B”, Policlinico Umberto I Hospital, Sapienza University of Rome, Viale regina elena 324, Rome, Italy.

\author{
Article Details \\ Article Type: Case Report \\ Received date: $03^{\text {rd }}$ April, 2020 \\ Accepted date: $29^{\text {th }}$ April, 2020 \\ Published date: $01^{\text {st }}$ May, 2020
}

"Corresponding Author: Alain J. Gelibter, Division of medical Oncology "B", Policlinico Umberto I Hospital, Sapienza University of Rome, Viale regina elena 324, Rome, Italy. E-mail: alain.gelibter@uniroma1.it

Citation: Gelibter, A.J., Siringo, M., Napoli, V.M., Cortesi, E., \& Marchetti, P. (2020). Case Report: ALK traslocated Pulmonary sarcomatoid carcinoma. J Case Reports Cancer 1(1):102. doi: https://doi.org/10.33790/jcrc1100102.

Copyright: (2020, This is an open-access article distributed under the terms of the Creative Commons Attribution License 4.0, which permits unrestricted use, distribution, and reproduction in any medium, provided the original author and source are credited.

\begin{abstract}
Introduction: Pulmonary sarcomatoid carcinoma (PSC) is a heterogeneous group of primitive lung cancer representing from $0.3 \%$ to $3 \%$ of all primary lung malignancies. Most patients with PSC consist of elderly, smoking men with a large, peripheral mass with welldefined margins.
\end{abstract}

Case Report: We report a case of carcinosarcoma with ALK translocation in a 43 years old woman with a good response to therapy with Alectinib and a Progression Free survival (PFS) of 15 months. She underwent Total body CT scan that showed bone, liver and spleen metastasis.

Histological sample was obtained with bronchoscopy, showing carcinosarcoma. Molecular analysis performed on tumor tissue showed an $80 \%$ immunochemistry ALK positivity and a negativity for EGFR, ROS 1 with a tumor proportion score (TPS) of programmed death ligand (PDL-1) of $20 \%$.

After one cycle of platinum-based doublet chemotherapy, interrupted for hematological toxicity, she was treated with Alectinib. We obtained a complete radiological response (CR) in extrapulmonary sites of disease but a SUV increased level was present in right upper lobe lesion (RUL). After few months she received Stereotactic Body Radiation Therapy (SBRT) in RUL showing a partial response.

Discussion: PSC are a mixture of carcinoma and sarcoma containing differentiated sarcomatous elements. Generally, they are considered chemo resistant. In the last years, there has been development of targeted and immunotherapy in lung cancer but the role of these drugs in PCS is still unknown. Lots of studies are evaluating the role of MET amplification, PDL1 overexpression, EGFR mutation, ALK translocation.

Conclusion: ALK rearrangement is a rare finding in PSC. This article shows the importance to test patients in order to find driver mutation even in rare and poor prognosis histological features of lung cancer.

Keywords: Sarcomatoid carcinoma (SC); lung; ALK, ALectinib

\section{Introduction}

Pulmonary sarcomatoid carcinoma (PSC) is a heterogeneous group of primitive lung cancer representing from $0.3 \%$ to $3 \%$ of all primary lung malignancies. According to the most recent 2015 World Health Organization (WHO) classification, PSC includes several types of malignant epithelial tumors (carcinomas) histologically mimicking sarcomas, showing or entirely lacking, a conventional component of non-small cell lung cancer (NSCLC). Thus, this rare group of lung neoplasms includes pleomorphic carcinoma, spindle cell carcinoma, giant cell carcinoma, pulmonary blastoma, and carcinosarcoma [1].
Most patients with PSC consist of elderly, smoking men with a large, peripheral mass with well-defined margins. As expected, PSC may pose diagnostic problems and immunohistochemistry is largely used [2]. Indeed, PSC tends to overexpress molecules associated with the epithelial-to-mesenchymal transition, such as vimentin, but the panel of immunostains also includes epithelial markers (cytokeratins, EMA), TTF-1, p40 and negative markers (e.g., melanocytic, mesothelial and sarcoma-related primary antibodies).

Although rare, PSC has increased their interest among oncologist community for different reasons: identification of the epithelialto-mesenchymal phenomenon as a major mechanism of secondary resistance to tyrosine kinase inhibitors; over-expression of PDL1 and effective treatment with immunotherapy; identification of c-MET exon 14 skipping mutation representing an effective target to Crizotinib and other specific inhibitors [3, 4].

The occurrence of pulmonary carcinomas showing a sarcomatous cell component is a well-known phenomenon indicating a divergent tumor cell dedifferentiation from epithelial to mesenchymal phenotype in conventional non-small cell lung cancer (NSCLC) [5]. In our case report we will see a rare case of PSC with ALK translocation and optimum response to ALK inhibitors.

\section{Case Report}

A 43 years old woman, good general condition at diagnosis. Karnowski performance status (KPS) $100 \%$ and eastern cooperative oncology group (ECOG) 0 . Not important disease described in previous medical history.

On July 2018 patient began with 2 weeks of no responsive cough to symptomatic therapy. Due to persistent cough she underwent to a chest $\mathrm{X}$ ray, which showed a suspected opacity area in the right upper lobe. Due to the imaging deriving from chest X Ray, she received a chest $\mathrm{CT}$ scan that showed a $5 \mathrm{~cm}$ solid lung lesion in the right upper lobe. Enlarging the field the CT scan revealed a $2 \mathrm{~cm}$ solid lesion in the $\mathrm{V}$ liver segment too. Due to radiological finding arising from CT scan, and in order to give the patient the correct staging, on 25th of July patient underwent to a 18 FDG Pet Scan that revealed an increased level of standard uptake values (SUV) in the right lung upper lobe (SUV 35), in spleen, left iliac bone, fifth liver segment and Dorsal vertebra (D3).

On August 2018 cito/histological sample was obtained with rigid bronchoscopy, showing typical feature of primitive lung neoplasia with mesenchymal differentiation like carcinosarcoma. Molecular analysis performed on tumor tissue showed an $80 \%$ immunochemistry ALK positivity and a negativity for EGFR, ROS 1 with a tumor proportion score (TPS) of programmed death ligand (PDL-1) of 20. 
In another hospital patient received single cycle of chemotherapy with Carboplatin AUC 5 (Day 1 q 21day) plus Gemcitabine $1000 \mathrm{mg}$ / $\mathrm{m} 2$ (Day 1-8 q 21 day) then aborted for a non-specified hematological G4 toxicity. Due to ALK -positivity and to chemotherapy toxicity in October 2018, Alectinib full dose treatment was started with a daily dose of $1200 \mathrm{mg}$ a day.

The treatment went on for 3 months with good tolerance and no relevant toxicities in patient reported outcomes. Not hematologic toxicities were described too. After only 2-3 weeks of treatment patient revealed an impressive cough improvement maintaining an optimal quality of life. The 18 FDG Pet- CT scan performed in January 2019, after three months of treatment showed a complete radiological response on spleen, left iliac bone, liver and D3, and a partial response (RECIST criteria) on pulmonary lesion. Cough completely disappeared and no toxicities appeared during the first three months treatment with Alectinib full dose.

After the first radiological evaluation patients continued with Alectinib treatment with no schedule modification. On May 201918
FDG Pet/CT scan confirmed a complete radiological response (CR) in extrapulmonary sites of disease but a SUV increased level was present in right upper lobe lesion (18 vs 7) while patients was still mantling her clinical benefit. Case has been discussed in our disease management team (DMT) weekly meeting. We decided to continue with Alectinib treatment while giving a radiotherapic consolidation treatment. Hematological test performed on May 2019 showed increased level of serum total bilirubin $(2.5 \mathrm{mg} / \mathrm{dl})$ with an upper limit normal value of laboratory of $1.5 \mathrm{mg} / \mathrm{dl}$. After Alectinib treatment interruption for 7 days, bilirubin serum level come back to normal range. On July 20192 days before starting radiation treatment We interrupted Alectinib treatment until a full dose of radiation therapy was given. A total 40 Gy treatment with Stereotactic Body Radiation Therapy (SBRT) was given between July 12, 2019 and July 27, 2019. On July 29th, 2019 Alectinib treatment was started again. PET scan performed in November 2019 showed a maintained radiological complete response in extrapulmonary sites of disease and a further radiological response in pulmonary right upper lobe.

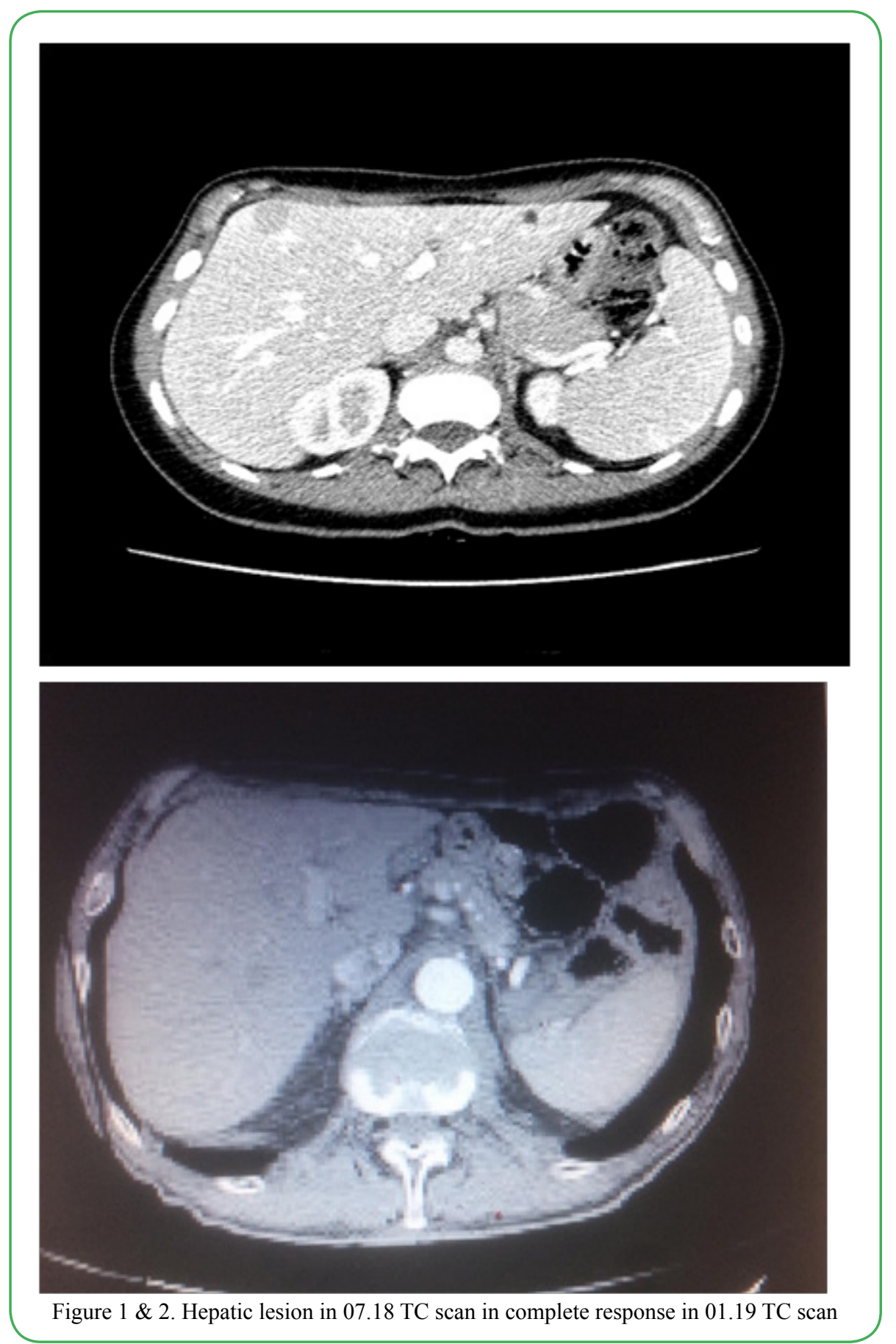




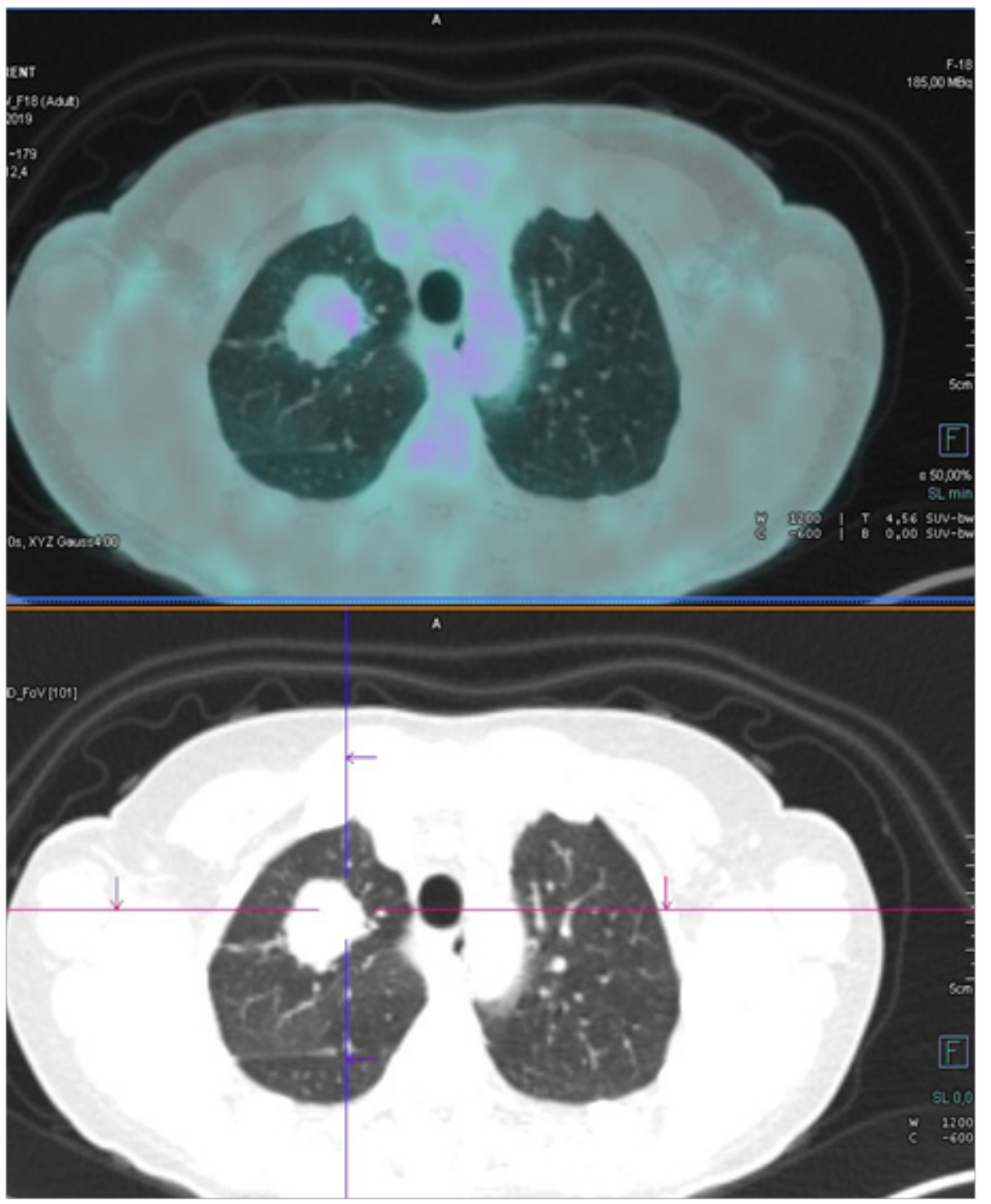

Figure $3 \& 4.5 \mathrm{~cm}$ solid lung lesion in the right upper lobe and partial response in $01.19 \mathrm{Pet} \mathrm{Tc}$ scan

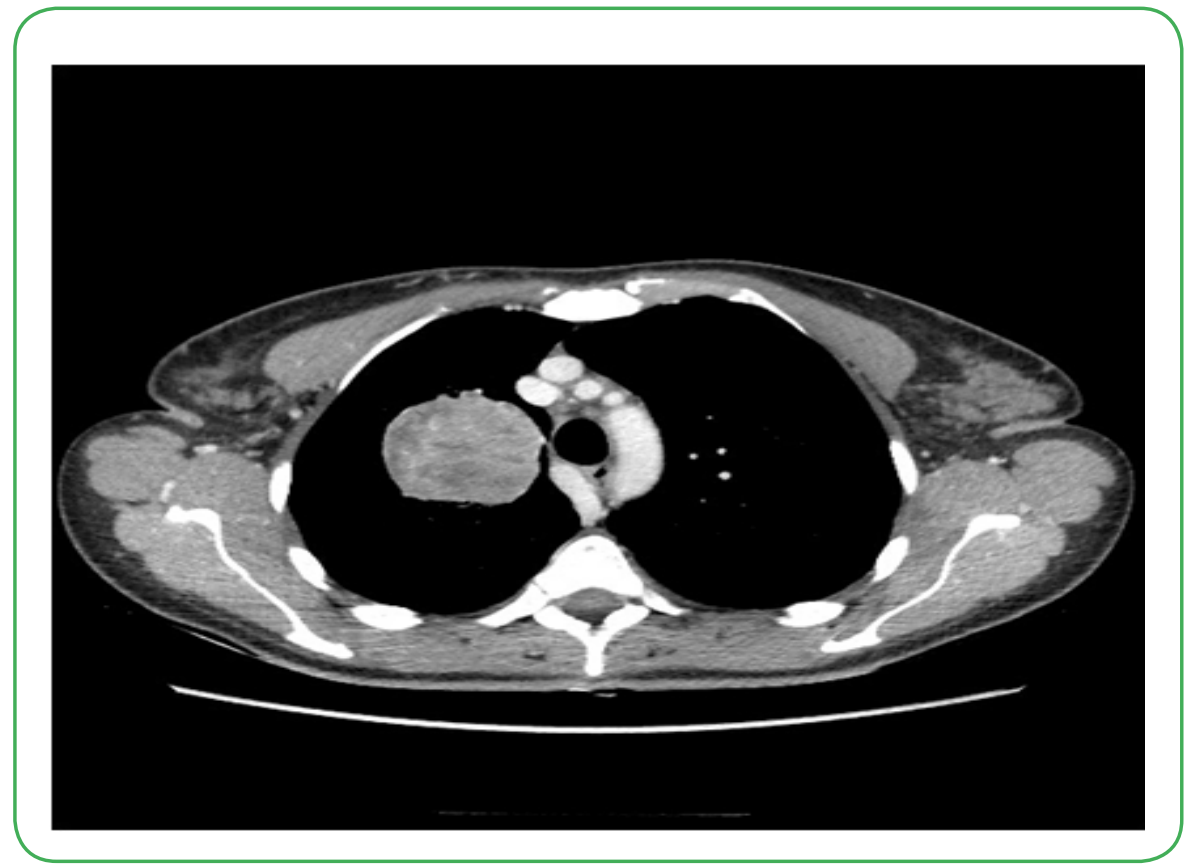




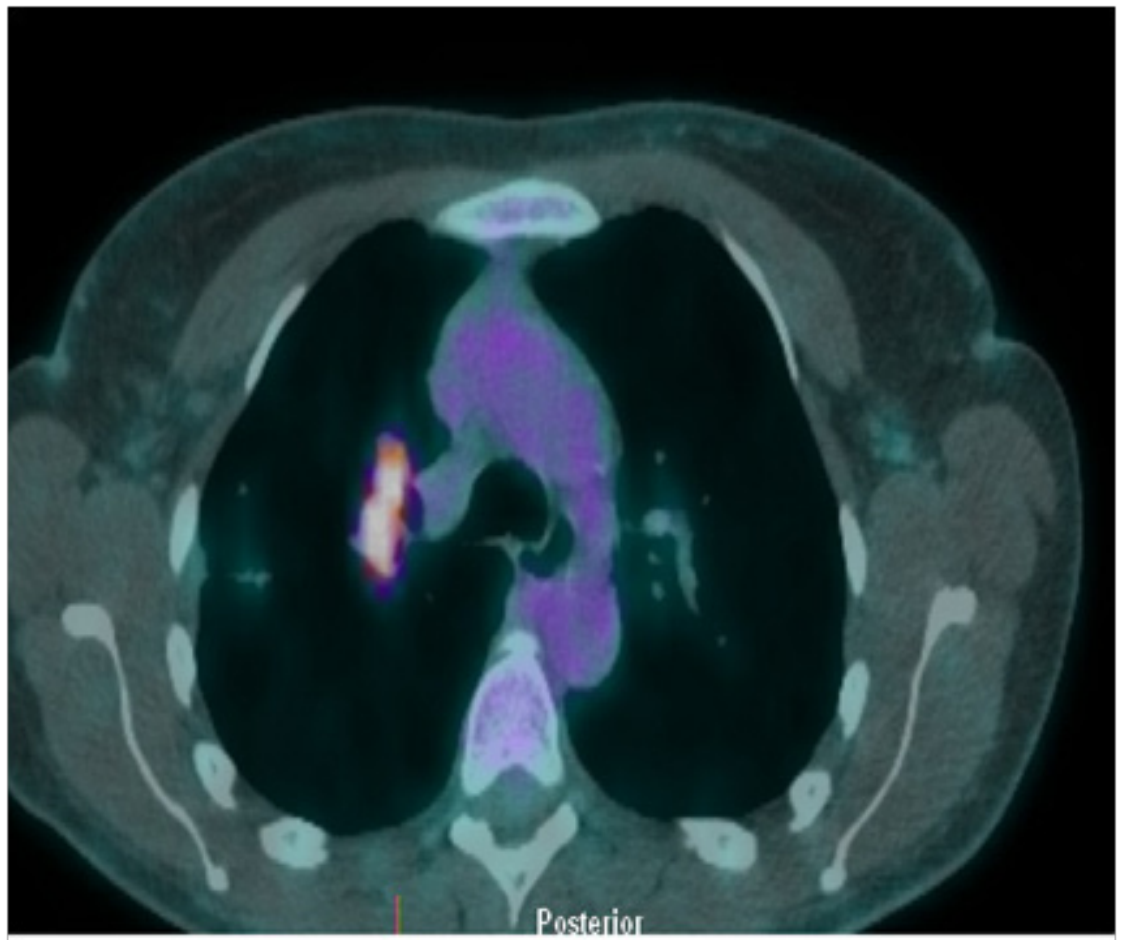

Figure 5. Dimensional decreasement in RUL from $18 \mathrm{~mm}$ to $7 \mathrm{~mm}$ and concomitant increasement in SUV in Pet scan of May 2019

\section{Discussion}

Carcinosarcoma is a rare malignant tumor with a mixture of carcinoma and sarcoma containing differentiated sarcomatous elements. Squamous cell carcinoma is the most common type of epithelial cell histology, followed by adenocarcinoma and adenosquamous carcinoma, whereas the mesenchymal component is most frequently rhabdomyosarcoma, followed by osteosarcoma and chondrosarcoma $[1,6,7]$.

Generally, lung carcinosarcomas (PSC) are treated with chemotherapy like NSCLC, usually with platinum-based doublet chemotherapy but they are considered often as chemo resistant and difficult to treat because our understanding for this disease is derived mostly from small retrospective studies and case series $[6,8]$.

The management of PSC is still unclear, we need new therapies to be more effective, so it's increased the interest in the identification of driver genomic alteration in PSC. For example, the recent discovery of frequent and actionable MET exon 14 skipping mutation can give us information about PCS biology and biomarker driven targeted therapy $[4,6,9]$.

In the last years, there has been development of targeted and immunotherapy in the lung cancer, which has improved the survival outcomes. Mostly epithermal growth factor receptor (EGFR), anaplastic lymphoma kinase (ALK), programmed death-1 (PD-1)and programmed death-ligand 1 (PD-L1)-targeted therapies have a promising therapeutic effect in patients with NSCLC but the potential clinical effect of these drugs to carcinosarcoma is still unknown.

There are lot of study demonstrating the potential clinical effects of target therapy in metastatic PCS: Velcheti et al. showed that PCS have higher PD-L1 levels than NSCLC, analyzing two large retrospective lung cancer cohorts, where 9 of 13 patients with PCS were positive for PD-L1 (15); Italiano et al studied that the overexpression of EGFR protein and high rate of KRAS mutation could be the reason for poor prognosis compared to other types of NCSLC [10-12]. Camidge et al. suggest that patients with high level of MET amplification may benefit from treatment with Crizotinib, a tyrosine kinase inhibitor of ALK/MET/ROS1 [4].
We reported a case of an ALK-positive carcinosarcoma with a good response to therapy with Alectinib, a second generation anti ALK drug approved in first line metastatic ALK positive NSCLC [13-15]. According to Chen et al. study, the incidence of ALK rearrangement in PSC is similar to other subtypes of NSCLC and more often among young patients with no smoking history. They have also reported a case of ALK-positive pleomorphic carcinoma with partial response to Crizotinib [16]. Pelosi et al studied that ALK gene amplification is associated with chromosome 7 (EGFR) and chromosome 17 (HER2) polysomy and suggested that ALK amplification represented an early, nonrandom and clonally related event in the carcinogenesis of PSC [17].

We didn't find in literature other case report treating Alectinib in PCS. Anyway, the role of molecular targeted therapy for this entity still needs further research and whenever possible all histological types of NSCLC should be investigated for this mutation.

\section{Conclusion}

This case report shows how even rare and poor prognosis histological features of lung cancer with driver molecular mutations can benefit from Alectinib treatment. Our patient is on treatment with Alectinib since October 2018 with a Progression Free Survival (PFS) of 15 months. This article shows the importance to test patients in order to find driver mutation even in rare and poor prognosis histological features of lung cancer.

Conflicts of Interest: The authors declare no conflicts of interest.

\section{References}

1. Travis, W.D., Brambilla, E., \& Nicholson, A.G, et al. The 2015 World Health Organization Classification of Lung Tumors.

2. Mochizuki, T., Ishii, G., \& Nagai, K, et al. (2008). Pleomorphic carcinoma of the lung: clinicopathologic characteristics of 70 cases. Am J Surg Pathol 32:1727-1735.

3. Pelosi, G., Sonzogni, A., \& De Pas, T, et al. (2010). Review article: pulmonary sarcomatoid carcinomas: a practical overview. Int J Surg Pathol 18:103-120 
4. Camidge, D.R., Ou S-HI., \& Shapiro, G, et al. (2014). Efficacy and safety of crizotinib in patients with advanced c-METamplified non-small cell lung cancer (NSCLC). J Clin Oncol 32(suppl; abstr 8001):5s.

5. Yang, Han., Yongbin, Lin., and Ying, Liang. (2017). "Treatment of lung carcinosarcoma and other rare histologic subtypes of non-small cell lung cancer." Current treatment options in oncology 18.9: 54 .

6. Shum, E., Stuart, M., Borczuk, A., Wang, F., Cheng, H., \& Halmos, B. (2016). Recent advances in the management of pulmonary sarcomatoid carcinoma. Expert review of respiratory medicine, 10(4), 407-416.

7. Franks, T.J., Galvin, J.R. (2010). Sarcomatoid carcinoma of the lung: histologic criteria and common lesions in the differential diagnosis. Arch Pathol Lab Med 134:49-54

8. Vieira, T., Girard, N., Ung, M, et al. (2013). Efficacy of firstline chemotherapy in patients with advanced lung sarcomatoid carcinoma. J Thorac Oncol 8:1574

9. Liu, X., Jia, Y., Stoopler, M.B, et al. (2016). Next-generation sequencing of pulmonary sarcomatoid carcinoma reveals high frequency of actionable MET gene mutations. J Clin Oncol 34(8):794-802.

10. Italiano, A., Cortot, A.B., Ilie, M, et al. (2009). EGFR and KRAS status of primary sarcomatoid carcinomas of the lung: implications for anti-EGFR treatment of a rare lung malignancy. Int J Cancer 125:2479-82.

11. Terra, S.B., Jang, J.S., Bi, L, et al. (2016). Molecular characterization of pulmonary sarcomatoid carcinoma: analysis of 33 cases. Mod Pathol 29:824-31.
12. Velcheti, V., Rimm, D.L., \& Schalper, K.A. (2013). Sarcomatoid lung carcinomas show high levels of programmed death ligand-1 (PD-L1). J Thorac Oncol 8:803-5.

13. Hida, T., Nokihara, H., Kondo, M., Kim, Y. H., Azuma, K., Seto, T., ... \& Hotta, K. (2017). Alectinib versus crizotinib in patients with ALK-positive non-small-cell lung cancer (J-ALEX): an open-label, randomised phase 3 trial. The Lancet, 390(10089), 29-39.

14. Peters, S., Camidge, D. R., Shaw, A. T., Gadgeel, S., Ahn, J. S., Kim, D. W., ... \& Zeaiter, A. (2017). Alectinib versus crizotinib in untreated ALK-positive non-small-cell lung cancer. New England Journal of Medicine, 377(9), 829-838.

15. Zhou, C., Kim, S. W., Reungwetwattana, T., Zhou, J., Zhang, Y., He, J., ... \& Xu, T. (2019). Alectinib versus crizotinib in untreated Asian patients with anaplastic lymphoma kinasepositive non-small-cell lung cancer (ALESIA): a randomised phase 3 study. The Lancet Respiratory Medicine, 7(5), 437-446.

16. Chen, X., Liang, J., Lu, J, et al. (2016). Pulmonary Sarcomatoid Carcinoma with ALK rearrangement: Frequency, ClinicalPathological Characteristics, and Response to ALK inhibitor. J Clin Oncol 34:15_suppl:e20055.

17. Pelosi, G., Gasparini, P., Cavazza, A, et al. (2012). Multiparametric molecular characterization of pulmonary sarcomatoid carcinoma reveals a nonrandom amplification of anaplastic lymphoma kinase (ALK) gene. Lung Cancer 77:507514. 